\title{
Mindfulness-Based Interventions for Schizophrenia
}

\author{
Contributors: , Ricardo M. Hodann-caudevilla ${ }^{1}$, Cintia Díaz-silveira ${ }^{2}$, Francisco \\ Alfonso Burgos-julián ${ }^{3}$, Miguel Angel Santed ${ }^{4}$ \\ 1, psyinvgroup@gmail.com \\ 2, Universidad Rey Juan Carlos. Facultad de Ciencias de la Salud. c/ Atenas, s/n. Alcorcón \\ (Madrid); cintia.diazsilveira@urjc.es \\ 3, Universidad Nacional de Educación a Distancia; psico.fburgos@gmail.com \\ 4, Universidad Nacional de Educación a Distancia; msanted@psi.uned.es
}

Version received: 13 July 2020

check for updates

Mindfulness-based interventions (MBIs) consist of short and structured intervention protocols focused on teaching patients the use of mindfulness for therapeutic purposes. Over the last three decades, there has been a remarkable expansion of this kind of interventions for the treatment of various psychopathological conditions. Despite this, MBls have only recently begun to be applied in the treatment of people with schizophrenia. Analysis of the available evidence on MBIs for the treatment of people with this disorder suggests that they are effective and safe treatments, although more research is needed to draw more robust conclusions.

\section{Definition}

The term mindfulness refers to a state of consciousness characterized by detached observation of the objects being attended to, in which equanimity and acceptance predominate. This state is achieved through metacognitive exercises in which attention is focused on the experience of the present moment ${ }^{[1]}$. Mindfulness mainly comes from the vipassana meditation of Buddhist tradition [2]. However, it was introduced in the Western world with a secular approach, as a technique oriented toward promoting the quality of life of people with high stress levels ${ }^{[3]}$. Over the years, mindfulness has been extended for therapeutic purposes to other health conditions, mainly due to its beneficial effect on people who present psychopathological profiles and somatic diseases ${ }^{4]}$. This expansion has taken place through the implementation of several intervention protocols included under the umbrella term "mindfulness-based interventions" (MBIs).

\section{Introduction}

Amongst the MBIs one can find two seminal protocols , Mindfulness-Based Stress Reduction (MBSR) ${ }^{[3]}$ and Mindfulness-Based Cognitive Therapy (MBCT) ${ }^{[5]}$. Both are widely used and have remarkable empirical support $[6][7]$. There is also a broad set of protocols that have been developed from MBSR and MBCT protocols for specific therapeutic purposes. Such protocols usually introduce variations regarding MBSR and MBCT in aspects such as program structure or pedagogical content. However, all MBls have, as a common point, the systematic training in meditation practices for the development of mindfulness, which is at the heart of the intervention [8]. Other distinctive aspects of MBIs have to do with the way they are administered: usually in group format and through one or more teachers who are not necessarily therapists or mental health professionals [9]. These aspects differentiate MBIs from other therapies-the so-called acceptance-based approaches_-in which mindfulness is used as a tool to promote psychological acceptance, but no systematic use of meditation practices is made [10]. This is the case with the Acceptance and Commitment Therapy (ACT) ${ }^{[11]}$ or the Dialectic Behavioral Therapy (DBT) ${ }^{[12]}$, in which mindfulness is taught primarily using short exercises and informal practices which consist of daily activities with a focus on the present moment.

The popularity of MBIs has increased exponentially over the last 30 years, alongside the volume of research conducted on their effectiveness in the treatment of different psychological conditions [13]. Nevertheless, evidence regarding their applicability in schizophrenia is still scarce. This is possibly partly due to a cautious reaction to a few 
early research papers, which found a relationship between meditation practice and the emergence of psychotic-type symptoms [14][15]. These publications reported that extended periods of meditation practiced over several consecutive days, in combination with other factors-a history of previous psychotic episodes, sleep deprivation, or the discontinuation of psychiatric medication-could lead to the onset of psychotic disorders. In this regard, authors such as Kuijpers et al. [16] have suggested that, under these conditions, meditation may act as a trigger of symptoms in individuals who have shown vulnerability. Despite this, some studies published in the early 2000 s found that the application of therapies which included mindfulness practice resulted in improvements in patients with schizophrenia [17]. Soon after, Chadwick ${ }^{[18]}$ developed the Person-based Cognitive Therapy for distressing psychosis (PBCT), the first intervention specifically designed for people with schizophrenia to incorporate mindfulness as a central element. In PBCT the treatment is based on a combination of therapeutic elements from cognitive models and different mindfulness practices, emphasizing a relationship with the patient based on acceptance [18].

\section{Latest Research}

Until March 2020, four meta-analyses have been published to date on the effectiveness of interventions, which include elements of mindfulness for people with psychosis: Khoury et al. [19], with 13 studies; Cramer et al. [20], with 8 studies; Louise et al. [21], with 10 studies; and Jansen et al. ${ }^{[22]}$, with 16 studies. It is noteworthy that their results are, in general, heterogeneous. Thus, only one reported large effects on overall symptomology [22], and two reported small effects [20][21]. Regarding the characteristic symptoms of schizophrenia, only one found moderate effects on positive symptoms $[20]$ and two found modest effects on negative symptoms ${ }^{[19][22]}$. However, it is relevant to note that the four meta-analyses cited included in their analyses both MBI's and acceptance-based approaches (ACT or DBT), which, as mentioned, have significant differences between them. They all included studies in which participants had psychotic symptoms but not necessarily a diagnosis of schizophrenia spectrum disorder. These methodological aspects provide a plausible explanation for the observed variability in their respective results.

The authors of this text conducted a systematic review and meta-analysis of randomised controlled trials (RCTs) focused on the application of MBls to people with schizophrenia, published up to the 25th of March 2020 [23]. The analysis of the 10 studies collected suggests that MBIs combined with standard interventions are able to generate significant improvements in a variety of clinical schizophrenia-related parameters, such as the intensity of overall symptomatology $(g=0.72)$, positive symptoms $(g=0.32)$, negative symptoms $(g=0.40)$, functioning level $(g=1.28)$, and awareness of illness $(g=0.65)$.

\section{Applications and Influences}

Current evidence suggests that MBls are safe for people with schizophrenia, at least when applied through structured protocols, in group settings and short-term practices. Furthermore, evidence suggests that they are effective treatments for people with schizophrenia spectrum disorders when used as adjuvant to TAU. As a consequence, they may in the near future become one of the chosen psychosocial approaches to treat people with psychosis, together with the interventions currently recommended in clinical guides.

However, it is also clear that the empirical evidence available at present is scarce; it is, therefore, necessary to conduct further studies that meet quality methodological standards in order to draw sound conclusions on the effectiveness of MBls. The results so far are promising.

\section{References}

1. Santed, M.Á. Procesos psicológicos en mindfulness. In Mindfulness: Fundamentos y Aplicaciones; Santed,M.Á., Segovia, S., Eds.; Paraninfo: Madrid, Spain, 2018; pp. 21-56.

2. Hanh, T.N.; Kornfield, J. Being Peace, 2nd ed.; Parallax Press: Berkeley, CA, USA, 2005. ISBN 978-1-888375-40-4.

3. Kabat-Zinn, J. An outpatient program in behavioral medicine for chronic pain patients based on thepractice of mindfulness meditation: Theoretical considerations and preliminary results. Gen. Hosp.Psychiatry 1982, 4, 33-47, doi:10.1016/0163-8343(82)90026-3.

4. Santed, M.Á.; Segovia, S. Mindfulness: Fundamentos y Aplicaciones; Paraninfo: Madrid, Spain, 2018.

5. Segal, Z.V.; Mark, G.W.; John, D.T. Mindfulness-Based Cognitive Therapy for Depression: A New Approach toPreventing Relapse, 2nd ed.; Guilford Press: New York, NY, USA, 2012. ISBN 978-1-4625-0750-4.

6. de Vibe, M.; Bjørndal, A.; Fattah, S.; Dyrdal, G.M.; Halland, E.; Tanner-Smith, E.E. Mindfulness-based stressreduction (MBSR) for 
improving health, quality of life and social functioning in adults: A systematic reviewand meta-analysis. Campbell Syst. Rev. 2017, 13, 1-264, doi:10.4073/csr.2017.11.

7. Goldberg, S.B.; Tucker, R.P.; Greene, P.A.; Davidson, R.J.; Kearney, D.J.; Simpson, T.L. Mindfulness-basedcognitive therapy for the treatment of current depressive symptoms: A meta-analysis. Cogn. Behav. Ther.2019, 48, 445-462, doi:10.1080/16506073.2018.1556330.

8. Kabat-Zinn, J. Mindfulness-based interventions in context: Past, present, and future. Clin. Psychol. Sci. Pract.2003, 10, 144-156, doi:10.1093/clipsy.bpg016.

9. Crane, R.S.; Brewer, J.; Feldman, C.; Kabat-Zinn, J.; Santorelli, S.; Williams, J.M.G.; Kuyken, W. Whatdefines mindfulness-based programs? The warp and the weft. Psychol. Med. 2017, 47, 990-999,doi:10.1017/s0033291716003317.

10. Carmody, J. Eastern and Western Approaches to Mindfulness: Similarities, Differences, and Clinicallmplications. In The Wiley Blackwell Handbook of Mindfulness; le, A., Ngnoumen, C.T., Langer, E.J., Eds.; JohnWiley \& Sons: Chichester, UK, 2014. ISBN 9781-11829-489-5.

11. Hayes, S.C.; Strosahl, K.D.; Wilson, K.G. Acceptance and Commitment Therapy: An Experiential Approach toBehavior Change; Guilford Press: New York, NY, USA, 1999. ISBN 978-1-57230-481-9.

12. Linehan, M. Cognitive-Behavioral Treatment of Borderline Personality Disorder, 1st ed.; The Guilford Press: NewYork, NY, USA, 1993. ISBN 978-0-89862-183-9.

13. Goldberg, S.B.; Tucker, R.P.; Greene, P.A.; Davidson, R.J.; Wampold, B.E.; Kearney, D.J.; Simpson, T.L.Mindfulness-based interventions for psychiatric disorders: A systematic review and meta-analysis. Clin.Psychol. Rev. 2017, 59, 52-60.

14. Epstein, M.D.; Lieff, J.D. Psychiatric complications of meditation practice. J. Transpers. Psychol. 1981, 13,137-147.

15. Walsh, R.; Roche, L. Precipitation of acute psychotic episodes by intensive meditation in individuals witha history of schizophrenia. Am. J. Psychiatry 1979, 136, 1085-1086, doi:10.1176/ajp.136.8.1085.

16. Kuijpers, H.J.; Van der Heijden, F.; Tuinier, S.; Verhoeven, W.M.A. Meditation-induced psychosis. Psychopathology 2007, 40, 461464,https://doi.org/10.1159/000108125.

17. Bach, P.; Hayes, S.C. The use of acceptance and commitment therapy to prevent the rehospitalization ofpsychotic patients: A randomized controlled trial. J. Consult. Clin. Psychol. 2002, 70, 1129-1139,doi:10.1037/0022-006x.70.5.1129.

18. Chadwick, P. Person-Based Cognitive Therapy for Distressing Psychosis; John Wiley \& Sons: Chichester, UK,2006. ISBN 0-47002984-6.

19. Khoury, B.; Lecomte, T.; Gaudiano, B.A.; Paquin, K. Mindfulness interventions for psychosis: A metaanalysis.Schizophr. Res. 2013, 150, 176-184,doi:10.1016/j.schres.2013.07.055.

20. Cramer, H.; Lauche, R.; Haller, H.; Langhorst, J.; Dobos, G. Mindfulness-and acceptance-basedinterventions for psychosis: A systematic review and meta-analysis. Glob. Adv. Health Med. 2016, 5, 30-43,doi:10.7453/gahmj.2015.083.

21. Louise, S.; Fitzpatrick, M.; Strauss, C.; Rossell, S.L.; Thomas, N. Mindfulness- and acceptance-basedinterventions for psychosis: Our current understanding and a meta-analysis. Schizophr. Res. 2018, 192, 57-63, doi:10.1016/j.schres.2017.05.023.

22. Jansen, J.E.; Gleeson, J.; Bendall, S.; Rice, S.; Alvarez-Jimenez, M. Acceptance- and mindfulness-basedinterventions for persons with psychosis: A systematic review and meta-analysis. Schizophr. Res. 2019,doi:10.1016/j.schres.2019.11.016.

23. Hodann-Caudevilla, R.M.; Díaz-Silveira, C.; Burgos-Julián, F.; Santed, M.Á. Mindfulness-based interventions for people with schizophrenia: A systematic review and meta-analysis. Int. J. Environ. Res. Public. Health 2020, 17, doi:https://doi.org/10.3390/ijerph17134690.

\section{Keywords}

mindfulness; effectiveness; schizophrenia; meta-analysis

(C) 2020 by the author(s). Distribute under a Creative Commans CC BY license 